\title{
Measuring Navigational Map Reading Competencies: A Pilot Study with a location-based GeoGame
}

\author{
Janina Bistron, Angela Schwering
}

\begin{abstract}
Navigational map reading (NMR) is relevant to people's everyday life, in professional contexts, and in school education. Being interested in fostering children's NMR competencies implies a test instrument for measuring potential learning progress. Related literature lacks an evaluated test for children that is focused on the spatial aspects of NMR and applicable at different locations. This paper fills this gap by presenting OriGami NMR Test - a test for measuring NMR competencies in children that is implemented in a digital geogame and played with a mobile device in the real-world. In order to enable a transfer of the test to different locations, we offer the reader the general concept of the test, the geogame, as well as a script that automatically scores the participants' test performances and evaluates the test items for the specific location. In a pilot study, we successfully realized and evaluated the test for two different locations.
\end{abstract}

Keywords: Navigational Map Reading, Map-based Orientation, Spatial Orientation, Paper Maps, Mobile GPS Devices, GeoGames, location-based Games, Test Instrument

\section{INTRODUCTION}

Orientation and navigation with maps have an important role in people's everyday life as well as in professional contexts (Hemmer et al. 2008). Both adults and children differ in their way and success in using the maps. These individual differences were observed in several studies for example: differences in navigation strategies of women and men (Dabbs et al. 1998) or in the map-based orientation among girls and boys or younger and older children (Hemmer et al. 2013). Considerable developmental changes in the understanding of maps take place at the age of 8 to 12 (Hemmer et al. 2013, Liben et al. 2007). However, the performance in using maps is not fixed to gender or age: Hemmer et al. (2013) studied more than 300 third to fifth graders and concluded that orientating with maps can be fostered via educational training. The results show a positive correlation between the children's experiences in using maps and their success in orientating with them. These outcomes underline the significance of maps in national school curricula and educational guidelines for primary or secondary schools from all over the world (e.g.: USA: NRC 2006, Germany: DGfG 2012, New Zealand: Ministry of Education NZ 2007, Hong Kong: CDC-HKEAA 2017).

Ishikawa et al. (2005) emphasize that training methods in geoscience education should be chosen according to the individual abilities of each student. This raises the question of which training medium can meet such individual needs. One answer could be found in geospatial technologies (like GPS): These technologies bear the potential to measure the user's movement in space and his or her interactions with the map as well as to react individually to it. In line with this argument, authors indicate the need of exploring this kind of technologies from an educational perspective (Baker et al. 2015). Moreover, studies emphasize people's preference to use digital navigation tools instead of paper maps (Hergan et al. 2017, Hurst et al. 2013, Speake et al. 2012) indicating that geospatial technologies found their way into many areas of 
people's lives. However, there is an evidence for negative short- and long-term effects of navigation tools on people's spatial knowledge, wayfinding, and orientation (Münzer et al. 2006, Field et al. 2011, Ishikawa 2019). This can be explained by the user's passivity when following navigation instructions (Ishikawa 2019). Nevertheless, we suspect that geospatial technologies can be adjusted for educational assessments and trainings by implementing cognitively-demanding tasks.

This research is on educational assessments with a special focus on training effects. The literature lacks an evaluated test that is created to be used by researchers or teachers for measuring the training effects on children's performances in navigating with maps. In this paper we will show how GI technologies can serve as a basis for such a test.

\section{Navigational Map Reading (NMR)}

In the scientific literature, there are different terms defining the activity of reading a map for orientation or navigation.

Hemmer et al. (2013) coin the term map-based (spatial) orientation (MBO) competence which refers to the transformation between map and reality. According to Ishikawa et al. (2005), three main understandings are involved in a map-reality transformation: The representational correspondence (understanding the meaning of the symbols), the configurational correspondence (finding the relation between a specific object and its representation on the map) and the directional correspondence (understanding the alignment of the map). MBO is defined as a competence which in turn is a broadly defined term in educational contexts: On the one hand, this term refers to the input (the attributes of people required to achieve a certain performance) and, on the other hand, it describes the output (result of training) in the sense of a "competent" performance (Hoffmann 1999). Hemmer et al. (2013) formulate a theoretical construct for the MBO competence by dividing the related process into the ability (a) to locate objects from the map in the real space and (b) vice versa, the abilities (c) in taking correct turns, (d) in pointing in directions correctly, (e) in estimating distances correctly as well as (f) in aligning the map according to the view direction while following a pre-defined route through a city center. Some of these abilities ask for map-to-real-world transformations, others for real-world-to-map transformations. The theoretical construct was revised factor analytically in a study with over 300 participating children. The results showed that tasks related to the above-mentioned abilities loaded onto one factor defined as the MBO competence.

Lobben (2007) uses the term navigational map reading (NMR) for describing navigation with a map to an unknown location. NMR is similarly defined to Hemmer's MBO competence and Hoffmann's understanding of competency: Associated with NMR tasks, Lobben defines different spatial abilities referring to people's aptitude (e.g. innate brain function) and achievements (through training and experience) in making spatial relations between objects in geographic space. In a study with 45 adults, she proved the predictive power of three of these abilities: (a) Self-location showed the highest predictive power followed by (b) route memory and - with an only weak significance - by (c) map rotation. The predictive power of these abilities was determined by correlating the participants' performance in corresponding paper pencil tests with their results in a real-world NMR performance test. Lobben (2007) studied selflocation by presenting participants a map and some photographs of different locations represented on the map. Using the photo, the subjects determined their location and view direction on the map. Route memory was investigated by showing the participants routes on maps and asking them to remember these routes by ordering verbal route descriptions in the right way. The map rotation ability was tested by presenting the participants two maps 
simultaneously and asking them to determine whether the second map was flipped or rotated. Since studies on map alignment reveal different "rotation strategies" depending on the way maps are presented and tasks are defined (Kozhevnikov et al. 2001, Aretz et al. 1992), we assume that Lobben's map-rotation task does not trigger the same strategies as NMR situations and therefore was not as predictive as expected. The real-world NMR performance test itself also included self-location and navigation tasks. It took place in a hallway network of a large building. The task performance was evaluated by considering the number of map rotations and hesitancies, the number and duration of stops, as well as the amount of time spent studying the map.

In our research approach, we will use the term navigational map reading (NMR) competencies in analogy with the definition of Lobben (2007). In accordance to Hemmer et al. (2013) we use the term "competencies" instead of "abilities".

\section{NMR Tests}

Being scientifically interested in children's NMR requires a test instrument that is able to measure children's learning progress. As shown above, some studies already exist that investigated NMR in different contexts, but the results of these studies do not fill this gap:

Hemmer et al. (2013) investigated influencing factors on children's MBO competence with a factor-analytically evaluated test, but did not create the test to be applicable in other settings. Lobben (2007) created a real-world map navigation exercise with an indoor set-up for validating NMR ability paper pencil tests with adult participants, but did not validate the real-world exercise itself, nor developed a test especially for children or real-world environments. Hergan et al. (2017) investigated the aided wayfinding of children by comparing navigation via mobile navigation and via paper-maps. Their focus is on comparing the performance in wayfinding using these tools, not on developing and evaluating a test for measuring children's NMR competencies.

In addition, the tests described are based on direct observations of the participants: External observers took notes on the participant's behavior and movement in space. Instead, studies on GPS data and trajectories reveal the potential of geospatial technologies for automatically collecting data on people's movements in space (e.g. Fu et al. 2016). This potential has not yet been exploited in the context of NMR so far.

Moreover, the tests described include not only configurational and directional aspects of NMR, but also representational transformations such as symbol or label reading. Symbol reading is often not the crux in navigating with a map (Lobben 2004) and label-reading must not be the crux, since we are not interested in reading skills when analyzing NMR situations. Therefore, an exclusively spatial NMR test is needed that offers all participants a common symbolunderstanding and does not request reading of street names or labels.

What is missing so far is an evaluated, digitally-supported real-world test for measuring children's NMR competencies with a focus on configurational and directional information which is applicable at different locations. The aim of our work is to offer a design for such a test and to show its potential by evaluating the test with a pilot data sample.

\section{OriGami NMR Test}

We developed a test for measuring NMR competencies in primary school children, which we call OriGami NMR Test. It aims to investigate the learning progress of NMR trainings and addresses configurational and directional transformations between the real-world and the 
map. The test is created for real-world set-ups and implemented in a location-based geogame called OriGami ("Orientation-Game") that displays the map, places the tasks, and collects data on the participants' movement in space and interactions with the map. With this technical foundation, we automatically measure, score, and evaluate the participants' performances in NMR tasks.

In this study, we evaluate OriGami NMR Test for two exemplary locations. To do so, we analyze the test items, estimate the reliability, and validate the total test scoring against an expert judgement.

OriGami NMR Test is also applicable at further locations: We offer the reader a detailed description of the test design and provide open access to OriGami for creating and conducting the test as well as to an evaluation script for calculating test scores and analyzing the data. 


\section{METHOD}

\section{Participants}

Fifty-one primary school students ( 29 boys and 22 girls) participated in the study. The mean age was 8.33 (sd: 1.11) years (range 6 to 10). The children were rewarded with a science-toy and a certificate of participation. We recruited the participants via letters handed out in local primary schools. The study was conducted as a summer event during the school vacations. We accepted primary school students who lived nearby and were familiar with the further or closer environment of the study area. We had no participant dropouts, but technical issues that made the data of one further participant useless.

\section{Materials}

\section{Study area}

The study areas were located in two different parks in North Rhine-Westphalia, Germany - the Citizens' Park in Senden and the Wildlife-Park in Coesfeld - each with short distances to walk. We chose these park areas to minimize the influence of urban structures on spatial NMR: The areas showed a branched network of paths and different objects for orientation (e.g. benches, trash cans, and trees). There were no street names and house numbers, but a lot of repetitive objects. These characteristics prevented orientation by reading street names and house numbers or by recognizing unique landmarks. Instead, they triggered NMR that asks for the combination of several spatial hints for orientation and navigation.

\section{OriGami}

We created a location-based geogame named OriGami, which is an app for GPS enabled mobile devices (e.g. tablets and smartphones). The current version of OriGami is based on prototype work (Bartoschek et al. 2018). The app allows the user to create map-based games (e.g. thematical rallies, treasure hunt games, and NMR trainings) for a chosen location. We used OriGami to realize OriGami NMR Test for two different locations. With OriGami, we offer a tool for applying the test also at further locations.

\section{OriGami NMR Test}

With the help of OriGami, we realized the following test design which can be transferred to different locations:

OriGami NMR Test consists of four NMR task types which we classify into two groups: Location Tasks (12 tasks in total) and Direction Tasks (8 tasks in total) (fig. 1).

Location Tasks include:

- Self-location tasks (LOC): Marking the own location on the map.

- Navigation-to-a-flag tasks (LNV): Changing the own location by navigating to a flag presented on the map.

Direction Tasks include:

- Mark-view-direction tasks (DM): Marking the own view direction on the map.

- Adopt-view-direction tasks (DA): Changing the own view direction through turning by adopting a direction that is marked on the map. 


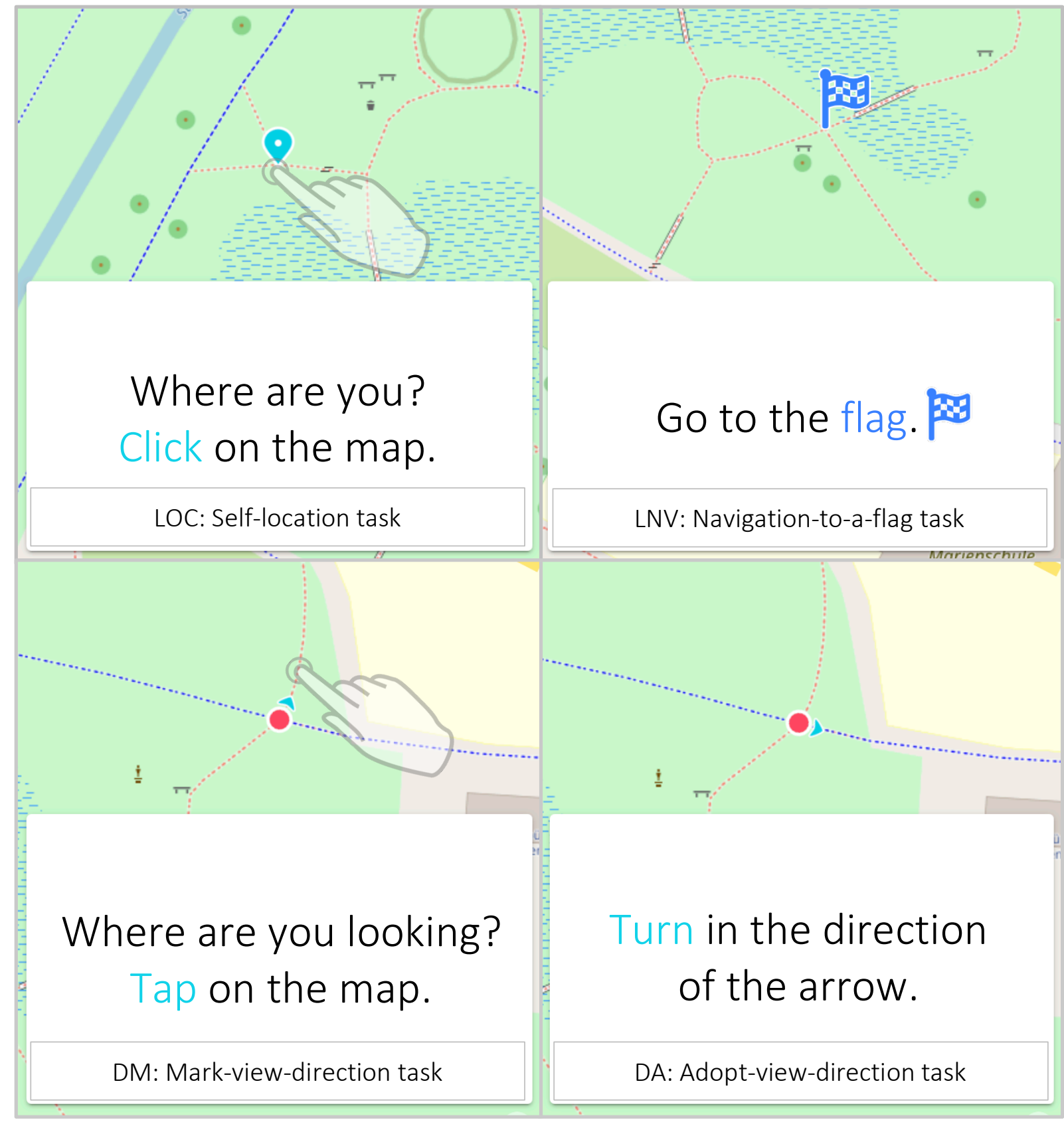

Figure 1: Location Tasks (LOC and LNV) and Direction Tasks (DM and DA) in OriGami NMR Test.

We chose tasks that represent the main activities of NMR situations following the work of Hemmer et al. (2013), Lobben (2007), and Ishikawa et al. (2005): With its Location and Direction Tasks, OriGami NMR Test represents the configurational and directional correspondences between map and real-world as mentioned by Ishikawa et al. (2005). In line with the real-world tests of Lobben (2007) and Hemmer et al. (2013), OriGami NMR Test consists of (self-)location and navigation tasks which we summarized with the term Location Tasks (LOC and LNV). Additionally, we created Direction Tasks to represent the direction-pointing tasks of Hemmer et al. (2013) in a way that is perceptible for a mobile device: We replaced the pointing task by a turn-around task (turn your body and the device) (DA tasks). LNV tasks ask for transformations from map to real-world and LOC tasks for transformations from real-world back to the map. DA tasks ask for map-to-real-world transformations. We added DM tasks in order to complete this task structure with Direction Tasks that also ask for real-world-to-map transformations. 
In total there are six LOC, six LNV, four DM, and four DA tasks. In OriGami NMR Test these tasks are presented in twins of two similar tasks having the same task difficulty. The tasks of each task twin:

- are of the same task type

- are located at comparable locations (i.e. the locations are comparable with respect to the surrounding objects and the path structures)

- $\quad$ are oriented in the same direction (i.e. the main view direction during or towards the tasks is the same)

Children show different competencies in solving NMR tasks depending on the angle between map alignment and view direction (Presson 1982). To ensure different task difficulties among the task twins - which is required in order to differentiate between strong and weak performances (Moosbrugger et al. 2020) - the tasks twins differ by the angle between map alignment (which is fixed to North) and view direction during or towards the task (which is north, south, or west/east). In order to avoid that the participants change the task difficulty by rotating the map, we forbid to rotate the device. Lobben (2007) interprets such physical map rotations as a criterion for a weaker NMR performance. Since we are not able to measure physical rotations with the sensors of the device (as we are not able to distinguish them from the participants' turning movements), we decided to forbid them and to measure the effects on the resulting task solution instead.

The tasks of OriGami NMR Test are placed in a sequence with so-called Transition Tasks leading from one task to the next one and ensuring a continuous gameplay. LOC tasks are always introduced with an arrow-navigation leading the children to the starting location without showing them the map. LNV tasks always start with the children's initial location marked on the map, which disappears when the flag appears. DM and DA tasks are introduced by arrownavigation and, additionally for DM tasks, by setting the view direction via turning the children into a certain direction. By creating some distance between the task locations, the Transition Tasks prevent knowledge transfer from one task to the next task.

Location- and direction-marker are disabled during the test and no automatic feedback on the accuracy of the task solution is given. Instead OriGami plays a sound after solving a task and shows the message "answer saved". The total game duration is around 30 to 45 minutes.

\section{Map material}

The digital map used in OriGami is based on standard OpenStreetMap material. Streets and paths are shown as colored lines, areas of grass, forests or water are colored in natural colors, houses are shown as polygons in their shape from top view, and objects like trees, statues, benches, bridges, or trash cans are represented with a symbol on the map. The test area is marked with a yellow line on the map. It is allowed to zoom and pan the map. At the beginning of each task, the app zooms out to the whole test area. At the end of each task it zooms into the task area (to the location- or direction-marker given by the task or set by the children) and asks the users to verify their given answer for a second time. This procedure ensures the same zoom level for all participants and prevents accidental responses.

\section{Video material}

A head-mounted camera filmed the children's speech and behavior. A screen-video recorded the gameplay in OriGami (incl. digital map, written task instructions, and markers on the map). 


\section{Experimental Design and Procedure}

\section{User Study}

We conducted OriGami NMR Test at two different locations. 29 participants took part in Senden and 22 took part in Dülmen. Each child was holding a tablet (with a 10.2" display) in their hand with OriGami installed and wore a camera mounted on their head. After a tutorial about the tablet usage, task types, and symbol meanings (to ensure a common basis for the representational correspondence of map representation and real world for all participants), the children started with OriGami NMR Test. During the test, the children were accompanied by the experimenter. The children were asked to think aloud explaining their ideas behind the solution. The children were allowed to take breaks and to ask questions about task instructions and symbols, but didn't received cues about solution strategies or explicit configurational or directional correspondences. Tasks were given in an easy and short written form. The children could also have the tasks read aloud to them using an audio button. All children participated alone without the influence of other participants. The study was done in compliance with the current Covid-19 regulations. The test performance of each participant was scored.

\section{Expertjudgement}

Three experts judged each child's NMR competencies on a scale from zero to ten based on the audio (think-aloud statements) and video material.

The experts were experienced student teachers recruited via job posting. They got payed for this task and signed a declaration of confidentiality. They had neither access to the OriGami data nor to the number of correct and incorrect solutions of each participant. However, they knew the correct solution from conducting the two tests themselves. They used the definition of NMR competencies described above and were asked to analyze every child in a wholistic way (i.e. without calculating total scores on the basis of the task solutions). We also informed the experts, that they were allowed to ignore individual tasks, if they think they were not relevant for evaluating the NMR competencies of the children.

The experts made their individual judgments and agreed together on one score for each child by discussing the individual cases.

\section{Data Analysis}

\section{Test Scoring}

OriGami automatically collects data on the participants' movements in space (location, speed, and view direction) and on their interaction with the tablet (tapping on the map, panning, and zooming), as well as timestamps and GPS accuracy data.

In order to score the participants' NMR competencies, we used data with the potential to describe the accuracy and the process in solving NMR tasks (tab. 1). The accuracy in solving a task is defined by the deviation (in $m$ or in degrees) from the correct solution. The process data gives additional information on the way a user solved a task. We assume that the number of pan and zoom interactions with the map (for LOC tasks), the length of the route walked (for LNV tasks), as well as the sum of turning movements (for DA tasks) grow with the uncertainty in solving these tasks. These assumptions follow the work of Hergan et al. (2017) and Lobben (2007) who define similar data for evaluating the NMR performance of their participants - e.g. the number of going "one crossroad too far", taking wrong turns, stops, spins around the own axis, hesitations, map-rotations, as well as the time in solving a task. 


\begin{tabular}{|c|c|c|c|}
\hline & & Accuracy Data & Process Data \\
\hline \multirow{2}{*}{$\begin{array}{c}\text { Location } \\
\text { Tasks }\end{array}$} & Self-location & $\begin{array}{c}\text { Distance (in m) between correct and } \\
\text { user's marked location. }\end{array}$ & $\begin{array}{l}\text { Number of pan and zoom } \\
\text { interactions with the map }\end{array}$ \\
\hline & Navigation-to-a-flag & $\begin{array}{c}\text { Distance (in } \mathrm{m} \text { ) between correct and } \\
\text { user's actual location. }\end{array}$ & $\begin{array}{l}\text { Route length walked during the task } \\
\text { (in m) }\end{array}$ \\
\hline \multirow{2}{*}{$\begin{array}{c}\text { Direction } \\
\text { Tasks }\end{array}$} & Mark-view-direction & $\begin{array}{l}\text { Deviation (in }{ }^{\circ} \text { ) between correct and } \\
\text { user's marked view direction. }\end{array}$ & 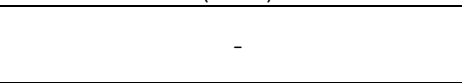 \\
\hline & Adopt-view-direction & $\begin{array}{c}\text { Deviation (in }{ }^{\circ} \text { ) between correct and } \\
\text { user's real view direction. }\end{array}$ & $\begin{array}{l}\text { Sum of turning movements while } \\
\text { solving the task (in }{ }^{\circ} \text { ) }\end{array}$ \\
\hline
\end{tabular}

Based on that data, we calculated an NMR competency score. Using the accuracy data, we scored one point for a correct solution. One additional point was obtained, if a correct answer was given in a pre-defined way: Tasks needed to be solved with small numbers of pan and zoom events, short route lengths (in comparison to the shortest correct solution of all participants) or a small sum of turning-angles.

We defined the thresholds (tab. 2) between the categories (two, one, or zero points per task) with data collected in test-runs in which we simulated different correct and incorrect answers with the help of several children and adults. Finally, we verified these thresholds with the test evaluation described below.

Table 2: Thresholds defining the categories in OriGami NMR Test.

\begin{tabular}{|c|c|c|c|}
\hline & & Thresholds for Accuracy Data & Thresholds for Process Data \\
\hline \multirow{2}{*}{$\begin{array}{l}\text { Location } \\
\text { Tasks }\end{array}$} & $\begin{array}{l}\text { Self- } \\
\text { location }\end{array}$ & $\begin{array}{l}\qquad d_{L O C}=10 \mathrm{~m} \\
d \text { is the deviation from the correct solution }\end{array}$ & $\begin{array}{l}n_{\text {pan }}=25 ; n_{\text {zoom }}=10 \\
n \text { is the number of events }\end{array}$ \\
\hline & $\begin{array}{l}\text { Navigation- } \\
\text { to-a-flag }\end{array}$ & $\begin{array}{l}\qquad d_{L N V}=5 \mathrm{~m} \\
d \text { is the deviation from the correct solution }\end{array}$ & $\begin{array}{c}2 \times \text { route }_{\min } \\
\text { route }_{\min } \text { is the shortest route to the correct destination } \\
\text { of all participants, determined for each LNV task } \\
\text { separately }\end{array}$ \\
\hline \multirow{2}{*}{$\begin{array}{l}\text { Direction } \\
\text { Tasks }\end{array}$} & $\begin{array}{l}\text { Mark-view- } \\
\text { direction }\end{array}$ & $\begin{array}{l}\qquad d_{D M}=25^{\circ} \\
d \text { is the deviation from the correct solution }\end{array}$ & - \\
\hline & $\begin{array}{l}\text { Adopt- } \\
\text { view- } \\
\text { direction }\end{array}$ & $\begin{array}{l}\qquad d_{D A}=25^{\circ} \\
d \text { is the deviation from the correct solution }\end{array}$ & $\qquad \begin{array}{c}\qquad \sum_{o}^{i} t_{i}=500^{\circ} \\
t_{i} \text { is the turning angle in }{ }^{\circ} \text {, determined by adding up } \\
i \text { turning angles }\end{array}$ \\
\hline
\end{tabular}

We were not interested in comparing absolute deviations (in $\mathrm{m}$ or in degrees) from the correct solutions because: (1) Smaller deviations from a correct solution might be induced by a bad GPS signal or the inaccuracy in marking locations and directions on the map and (2) wrong answers with larger deviations might not necessarily be worse than wrong answers with smaller ones (e.g.: a child could choose a wrong location in a LOC task in $200 \mathrm{~m}$ deviation from the correct solution which shows more similarities to the correct solution than any other solution with a deviation of less meters).

\section{Test evaluation}

We did a test evaluation based on the classical test theory (Moosbrugger et al. 2020): We analyzed the items via item difficulties and item-total correlations and estimated the reliability via Cronbach's Alpha and split-half reliability.

Moreover, we used the expert scorings for a test score validation. Following Moosbrugger et al. (2020), we understand validation as the process to collect and examine evidences for an 
intended interpretation of the test score. We want the OriGami NMR Test score to describe the spatial NMR competencies of children. We realized it by deciding on task types that were already successfully included or comparably present in similar tests. Moreover, we want the score to represent the NMR competencies from an educational or teacher's perspective, because in educational contexts the learning progresses of students are usually evaluated by teachers. These evaluations often happen in complex observation situations (e.g. based on think-aloud statements). As described above, the digital implementation of OriGami NMR Test allows an automatic performance evaluation instead. Therefore, by validating the OriGami NMR Test score against the expert scorings, we answered the following question: Is the automatically determined OriGami NMR Test score capable of reflecting a teacher's evaluation of children's spatial NMR competencies - with respect to the comparison of performances or to the learning progress of an individual child?

\section{Dimensions}

In analogy to the items of the test by Hemmer et al. (2013), the items of OriGami NMR Test should be unidimensional (i.e. describing one factor). The related cognitive processes behind the tasks support this assumption: Whereas Direction Tasks ask for directions, Location Tasks ask for locations derived from directions (e.g. from the own walking-direction, the current view direction, or the directional relations between objects). However, the number of participants was not sufficient for a factor analysis in order to check whether the individual items can be assigned to one or different factors (Bühner 2011). Unidimensionality is a precondition for calculating a total test score or estimating the reliability (Moosbrugger et al. 2020). Therefore, the OriGami NMR Test evaluation was done for (1.) all tasks (assuming one factor behind all tasks) and for (2.) the Location Tasks and Direction Tasks separately (assuming one factor behind each group of tasks).

Some of the participants panned and zoomed the map several times, some took long routes to the flag, and some turned around a lot of times before taking a decision for a view direction which is all together summarized in the process data. As mentioned above, we assume that the process data mainly describes the uncertainty in solving the individual task. However, in order to learn more about this aspect, a factor analysis is needed to show that the process data defines no own factor itself and can be used additionally to the accuracy data in order to evaluate the task performances. Therefore, the test evaluation was also done for (1.) the accuracy data only as well as (2.) for the accuracy and process data. 


\section{RESULTS}

\section{Difficulty of the test and individual test items}

We determined the difficulty indices $P_{i}$ of each item (task) $i$. As intended, the individual items show different difficulty indices spanning from $P_{L N V 4}=33$ (hardest task) to $P_{D A 4}=93$ (easiest task) for the location Senden and $P_{L N V 3}=23$ (hardest task) to $P_{D A 3}=91$ (easiest task) for the location Dülmen (tab. 3).

Based on the item difficulty indices, we built item twins and divided the test into two halves.

Table 3: Item difficulties $P_{i}$ (in brackets) and test-halves of OriGami NMR Test.

\begin{tabular}{|c|c|c|c|c|c|c|c|c|}
\hline Location & \multicolumn{2}{|l|}{ Senden } & & & \multicolumn{2}{|l|}{ Dülmen } & & \\
\hline \multirow[t]{2}{*}{ Data } & \multicolumn{2}{|c|}{ Only accuracy data } & \multicolumn{2}{|c|}{$\begin{array}{l}\text { Accuracy data } \\
\text { and process data }\end{array}$} & \multicolumn{2}{|c|}{ Only accuracy data } & \multicolumn{2}{|c|}{$\begin{array}{l}\text { Accuracy data } \\
\text { and process data }\end{array}$} \\
\hline & Test half 1 & Test half 2 & Test half 1 & Test half 2 & Test half 1 & Test half 2 & Test half 1 & Test half 2 \\
\hline \multirow{6}{*}{$\begin{array}{l}\text { Location } \\
\text { Tasks }\end{array}$} & LOC5 (41) & LOC2 (62) & LOC5 (40) & LOC2 (59) & LOC6 (36) & LOC5 (41) & LOC6 (36) & LOC3 (39) \\
\hline & LOC6 (69) & LOC3 (66) & LOC6 (67) & LOC3 (59) & LOC2 (55) & LOC3 (45) & LOC4 (50) & LOC5 (39) \\
\hline & LOC1 (76) & LOC4 (79) & LOC1 (71) & LOC4 (76) & LOC1 (59) & LOC4 (59) & LOC2 (55) & LOC1 (57) \\
\hline & LNV3 (62) & LNV4 (48) & LNV3 (50) & LNV4 (33) & LNV6 (50) & LNV3 (27) & LNV6 (30) & LNV3 (23) \\
\hline & LNV5 (62) & LNV6 (69) & LNV5 (52) & LNV6 (67) & LNV4 (55) & LNV1 (59) & LNV1 (48) & LNV4 (48) \\
\hline & LNV2 (90) & LNV1 (76) & LNV2 (76) & LNV1 (74) & LNV5 (68) & LNV2 (64) & LNV5 (64) & LNV2 (59) \\
\hline \multirow{4}{*}{$\begin{array}{l}\text { Direction } \\
\text { Tasks }\end{array}$} & DM2 (55) & DM1 (86) & DM2 (55) & DM1 (86) & DM4 (45) & DM2 (55) & DM4 (45) & DM2 (55) \\
\hline & DM3 (93) & DM4 (86) & DM3 (93) & DM4 (86) & DM3 (82) & DM1 (73) & DM3 (82) & DM1 (73) \\
\hline & DA1 (72) & DA3 (76) & DA1 (47) & DA3 (72) & DA2 (68) & DA1 (73) & DA1 (61) & $\mathrm{DA} 2(61)$ \\
\hline & DA4 (93) & DA2 (86) & DA4 (84) & DA2 (78) & DA3 (91) & DA4 (86) & DA3 (91) & DA4 (84) \\
\hline
\end{tabular}

\section{Item-total correlations}

Within the group of Location Tasks and the group of Direction Tasks, we determined item-total correlations with a part-whole-correction for each task. The majority of the items show a highly positive item-total correlation, but we also observe items with a correlation close to zero or with negative coefficients (tab. 4).

Table 4: Item-total correlations of OriGami NMR Test - item-total correlations lower than 0.3 are marked grey.

\begin{tabular}{|c|c|c|c|c|c|c|c|c|}
\hline Location & \multicolumn{2}{|l|}{ Senden } & & & \multicolumn{2}{|l|}{ Dülmen } & & \\
\hline \multirow[t]{2}{*}{ Data } & \multicolumn{2}{|c|}{ Only accuracy data } & \multicolumn{2}{|c|}{$\begin{array}{l}\text { Accuracy data } \\
\text { and process data }\end{array}$} & \multicolumn{2}{|c|}{ Only accuracy data } & \multicolumn{2}{|c|}{$\begin{array}{l}\text { Accuracy data } \\
\text { and process data }\end{array}$} \\
\hline & Test half 1 & Test half 2 & Test half 1 & Test half 2 & Test half 1 & Test half 2 & Test half 1 & Test half 2 \\
\hline \multirow{6}{*}{$\begin{array}{l}\text { Location } \\
\text { Tasks }\end{array}$} & LOC5 (0.30) & LOC2 (0.43) & LOC5 (0.29) & LOC2 (0.43) & LOC6 (0.63) & LOC5 (0.82) & LOC6 (0.66) & LOC3 $(0.72)$ \\
\hline & LOC6 (0.42) & LOC3 (0.39) & LOC6 $(0.47)$ & LOC3 (0.39) & LOC2 (0.47) & LOC3 $(0.51)$ & LOC4 (0.72) & LOC5 (0.88) \\
\hline & LOC1 (0.61) & LOC4 (0.81) & LOC1 (0.67) & LOC4 (0.71) & LOC1 (0.55) & LOC4 (0.76) & LOC2 (0.39) & LOC1 (0.49) \\
\hline & LNV3 (0.48) & LNV4 (0.53) & LNV3 (0.41) & LNV4 (0.62) & LNV6 (0.54) & LNV3 (0.74) & LNV6 (0.45) & LNV3 $(0.71)$ \\
\hline & LNV5 (0.65) & LNV6 (0.66) & LNV5 (0.66) & LNV6 (0.66) & LNV4(-0.06) & LNV1(-0.12) & LNV1(-0.25) & LNV4(-0.01) \\
\hline & LNV2 (0.68) & LNV1 (0.14) & LNV2 (0.75) & LNV1 (0.04) & LNV5 (0.16) & LNV2 (0.44) & LNV5 (0.23) & LNV2 (0.47) \\
\hline \multirow{4}{*}{$\begin{array}{l}\text { Direction } \\
\text { Tasks }\end{array}$} & DM2 (0.30) & DM1 (0.26) & DM2 (0.33) & DM1 (0.31) & DM4 (0.57) & DM2 (0.35) & DM4 (0.52) & DM2 (0.46) \\
\hline & DM3 (0.46) & DM4 (0.78) & DM3 (0.40) & DM4 (0.76) & DM3 (0.15) & DM1(-0.17) & DM3 (0.18) & DM1(-0.14) \\
\hline & DA1 (0.63) & DA3 $(0.83)$ & DA1 (0.58) & DA3 (0.82) & DA2 (0.15) & DA1 $(0.60)$ & DA1 (0.67) & DA2 (0.15) \\
\hline & DA4 (0.53) & DA2 (0.15) & DA4 (0.59) & DA2 (0.37) & DA3 $(0.64)$ & DA4 (0.61) & DA3 $(0.57)$ & DA4 (0.53) \\
\hline
\end{tabular}

Since low or negative item-total correlations can also be induced by the affiliation to different factors (Moosbrugger et al. 2020), we proceeded the further test evaluation in two different ways: (1.) We evaluated the test for all items and (2.) we also did the calculations by excluding tasks with an item-total correlation lower than 0.3. 


\section{OriGami NMR Test Score}

We defined the OriGami Test Score $O N M R_{v}$ for participant $v$ as the sum of achieved points. The test scale was defined from 0 to 20 points for considering the accuracy data (20 tasks with max. 1 point per task) and 0 to 40 for considering the accuracy and process data ( 20 tasks with max. 2 points per task). When focusing on Location Tasks only, the test scale was defined from 0 to 12 points for considering the accuracy data (12 tasks with max. 1 point per task) and 0 to 24 for considering the accuracy and process data (12 tasks with max. 2 points per task).

The actual scores for the 51 participants span different intervals of these possible total test scales: For the accuracy data, $O N M R_{v}$ ranges from 4 to 20 points (mean: 14.48 , sd: 4.28 , mode: 15, median: 16, quantiles Q1: 12, Q3: 17) for the location Senden and from 6 to 19 points (mean: 11.91, sd: 4.09, mode: 13, median: 12.5, quantiles Q1: 8.25, Q3: 15.5) for the location Dülmen. Considering accuracy and process data, $O N M R_{v}$ ranges from 6 to 40 points (mean: 26.48, sd: 8.44, mode: 20, median: 28, quantiles Q1: 22, Q3: 32) for the location Senden and from 11 to 36 points (mean: 21.95, sd: 7.85, mode: 33, median: 20.5, quantiles Q1: 15.5, Q3: 29.25) for the location Dülmen.

Considering only the accuracy data of the Location Tasks, $O N M R_{v}$ ranges from 2 to 12 points (mean: 8, sd: 2.99, mode: 9, median: 9, quantiles Q1: 6, Q3: 10) for the location Senden and from 1 to 11 points (mean: 6.18, sd: 2.91, mode: 7, median: 6.5, quantiles Q1: 4, Q3: 8.75) for the location Dülmen. Considering only the accuracy and process data of the Location Tasks, $O N M R_{v}$ ranges from 3 to 24 points (mean: 14.45, sd: 5.69, mode: 17, median: 16, quantiles Q1: 10, Q3: 18) for the location Senden and from 2 to 20 points (mean: 10.91, sd: 5.44, mode: 5, median: 10, quantiles Q1: 7.25, Q3: 15.75) for the location Dülmen.

Focusing on the accuracy and process data of all tasks except from the tasks with an item-total correlation lower than $0.3, O N M R_{v}$ ranges from 3 to 32 points (mean: 21.52, sd: 7.31, mode: 22, median: 23, quantiles Q1: 18, Q3: 27) for the location Senden and from 2 to 21 points (mean: 12.05, sd: 6.33, mode: 21, median: 11, quantiles Q1: 7.25, Q3: 18.5) for the location Dülmen.

To sum up, for the Location Senden the median is always slightly above the mean, i.e. $O N M R_{v}$ scores that are above the mean can be observed more frequent than scores below the mean. When considering accuracy and process data for the location Dülmen, the opposite is the case. This can also be observed in the skewness of the data distribution: The skewness for the accuracy data of Senden is -0.95 , for the accuracy and process data it is -0.77 . When considering only the Location Tasks, the skewness is -0.75 for the accuracy data -0.55 for the accuracy and process data. Excluding the tasks with an item-correlation of less than 0.3 leads to a skewness of -0.92 for the accuracy and process data of all tasks. For the location Dülmen, the skewness of the accuracy data is 0.12 , for the accuracy and process data it is 0.35 . When considering only the Location Tasks, the skewness is -0.01 for the accuracy data and 0.27 for the accuracy and process data. Excluding the tasks with an item-correlation of less than 0.3 leads to a skewness of 0.20 for the accuracy and process data of all tasks.

Zero points or very low scores were not reached by any participant. Instead, maximum scores were reached by a few for the location Senden, but not for the location Dülmen. The mean and the median of the Senden data are always slightly above the mean and median of the Dülmen data. 


\section{Reliability}

\section{Cronbach's Alpha}

For estimating the reliability, some preconditions need to be checked that are mainly based on a factor analysis. If the preconditions check is not possible (due to the small sample size - as in the case of this study), Moosbrugger et al. (2020) recommend to estimate the reliability via Cronbach's Alpha which gives even reasonably results when the preconditions are not met completely.

We estimated the Cronbach's Alpha for the Location and Direction Tasks separately. For the scoring of the accuracy data, Cronbach's Alpha is 0.78 for the Location Tasks and 0.66 for the Direction Tasks of Senden as well as 0.71 for the Location Tasks and 0.35 for the Direction Tasks of Dülmen. For the scoring of the accuracy and process data, Cronbach's Alpha is 0.78 for the Location Tasks and 0.70 for the Direction Tasks of Senden as well as 0.71 for the Location Tasks and 0.37 for the Direction Tasks of Dülmen.

We recognized up to three tasks for Senden and up to six tasks for Dülmen with an item-total correlation lower than 0.3. Dropping the related tasks improves Cronbach's Alpha for the accuracy data to $0.80(+0.02)$ for the Location Tasks and $0.70(+0.04)$ for the Direction Tasks of Senden as well as to $0.72(+0.01)$ for the Location Tasks of Dülmen. For the accuracy and process data, the dropping improves Cronbach's Alpha to $0.80(+0.02)$ for the Location tasks of Senden as well as to $0.72(+0.01)$ for the Location Tasks and $0.42(+0.05)$ for the Direction Tasks of Dülmen.

\section{Split-half reliability}

Despite even stricter preconditions, we decided to estimate the split-half reliability, since we are aiming for a test that is applicable to measure learning progresses: The two test-halves we built (tab. 3) are potential pre- and a posttest for such a measurement. We correlated the scorings of the test-halves and estimated the split-half reliability with the spearman-brown formula. For Senden and Dülmen, the split-half reliability values are higher than 0.8 and always higher than the corresponding Cronbach's Alphas (tab. 5).

Table 5: Split-half reliability of OriGami NMR Test.

\begin{tabular}{|l|l|l|l|l|}
\hline & \multicolumn{2}{|l|}{ Senden } & Dülmen \\
\hline & Accuracy Data & $\begin{array}{l}\text { Accuracy Data } \\
\text { \& Process Data }\end{array}$ & Accuracy Data & $\begin{array}{l}\text { Accuracy Data } \\
\text { \& Process Data }\end{array}$ \\
\hline $\begin{array}{l}\text { Location Tasks } \\
\text { excl. tasks with low } \\
\text { item-total correlation }\end{array}$ & 0.83 & 0.87 & 0.82 & 0.86 \\
\hline All tasks & 0.89 & 0.82 & & 0.92 \\
\hline $\begin{array}{l}\text { excl. tasks with low } \\
\text { item-total correlation }\end{array}$ & & 0.91 & 0.83 & 0.87 \\
\hline
\end{tabular}

\section{Validation against expert judgement}

We correlated the scorings of OriGami NMR Test with the scorings of the expert judgements. The determined correlation coefficients (in the following, simplified referred to as validity coefficients) range from 0.82 to 0.92 . They are at highest when including process data for the test scoring and at lowest when dropping tasks with an item-total correlation lower than 0.3 (tab. 6). 
Table 6: Correlations coefficients for ONMRv vs. the expert scoring.

\begin{tabular}{|l|l|l|l|l|}
\hline & \multicolumn{2}{|l|}{ Senden } & Dülmen \\
\hline & Accuracy Data & $\begin{array}{l}\text { Accuracy Data } \\
\text { \& Process Data }\end{array}$ & Accuracy Data & $\begin{array}{l}\text { Accuracy Data } \\
\text { \& Process Data }\end{array}$ \\
\hline $\begin{array}{l}\text { Location Tasks } \\
\text { excl. tasks with low } \\
\text { item-total correlation }\end{array}$ & 0.86 & 0.90 & 0.92 & 0.92 \\
\hline $\begin{array}{l}\text { All tasks } \\
\begin{array}{l}\text { excl. tasks with low } \\
\text { item-total correlation }\end{array}\end{array}$ & 0.90 & 0.85 & & 0.84 \\
\hline
\end{tabular}

\section{Sensitivity Analysis}

Knowing that the evaluation of OriGami NMR Test is affected by the pre-defined thresholds for the point-categories, we did a sensitivity analysis on different possible threshold combinations and its effect on the resulting test evaluation. In tab. 7 we present five examples. Details on the complete sensitivity analysis can be found in the corresponding R-Script that allows the reader to manipulate and analyze the thresholds on his or her own.

Table 7: Sensitivity Analysis - thresholds and their effect on the test evaluation (changes in the thresholds are marked in bold).

\begin{tabular}{|c|c|c|c|c|c|c|}
\hline \multirow{3}{*}{ Example } & \multicolumn{2}{|c|}{ Thresholds } & \multirow{2}{*}{\multicolumn{2}{|c|}{$\begin{array}{c}\text { Reliability value } \\
\text { All tasks } \\
\text { Accuracy \& Process Data }\end{array}$}} & \multirow{2}{*}{\multicolumn{2}{|c|}{$\begin{array}{l}\text { Validity coefficient } \\
\text { All tasks } \\
\text { Accuracy \& Process Data }\end{array}$}} \\
\hline & \multirow{2}{*}{ Accuracy Data } & \multirow{2}{*}{ Process Data } & & & & \\
\hline & & & Senden & Dülmen & Senden & Dülmen \\
\hline Initial & $\begin{array}{l}d_{L O C}=10 \mathrm{~m} \\
d_{L N V}=5 \mathrm{~m} \\
d_{D M}=25^{\circ} \\
d_{D A}=25^{\circ}\end{array}$ & $\begin{array}{l}n_{\text {pan }}=25 \\
n_{\text {zoom }}=10 \\
2 \times \text { route }_{\text {min }} \\
\sum_{o}^{i} t_{i}=500^{\circ}\end{array}$ & 0.91 & 0.87 & 0.91 & 0.89 \\
\hline 1 & $\begin{array}{l}\boldsymbol{d}_{L O C}=\mathbf{1 5} \mathbf{m} \\
d_{L N V}=5 \mathrm{~m} \\
d_{D M}=25^{\circ} \\
d_{D A}=25^{\circ}\end{array}$ & $\begin{array}{l}n_{\text {pan }}=25 \\
n_{\text {zoom }}=10 \\
2 \times \text { route }_{\min } \\
\sum_{0}^{i} t_{i}=500^{\circ}\end{array}$ & $0.90 \Downarrow$ & $0.82 \Downarrow$ & 0.91 & $0.91 \Uparrow$ \\
\hline 2 & $\begin{array}{l}d_{L O C}=10 \mathrm{~m} \\
\boldsymbol{d}_{L N V}=\mathbf{1 0 ~} \mathbf{m} \\
d_{D M}=25^{\circ} \\
d_{D A}=25^{\circ}\end{array}$ & $\begin{array}{l}n_{\text {pan }}=25 \\
n_{\text {zoom }}=10 \\
2 \times \text { route }_{\min } \\
\sum_{0}^{i} t_{i}=500^{\circ}\end{array}$ & 0.91 & $0.89 \Uparrow$ & $0.93 \Uparrow$ & 0.89 \\
\hline 3 & $\begin{array}{l}d_{L O C}=10 \mathrm{~m} \\
d_{L N V}=5 \mathrm{~m} \\
\boldsymbol{d}_{\boldsymbol{D M}}=\mathbf{3 0}^{\circ} \\
\boldsymbol{d}_{\boldsymbol{D A}}=\mathbf{3 0}^{\circ}\end{array}$ & $\begin{array}{l}n_{\text {pan }}=25 \\
n_{\text {zoom }}=10 \\
2 \times \text { route }_{\text {min }} \\
\sum_{o}^{i} t_{i}=500^{\circ}\end{array}$ & $0.90 \Downarrow$ & $0.79 \Downarrow$ & $0.92 \Uparrow$ & $0.90 \Uparrow$ \\
\hline 4 & $\begin{array}{l}d_{L O C}=10 \mathrm{~m} \\
d_{L N V}=5 \mathrm{~m} \\
d_{D M}=25^{\circ} \\
d_{D A}=25^{\circ}\end{array}$ & $\begin{array}{l}\boldsymbol{n}_{\text {pan }}=\mathbf{3 0} \\
\boldsymbol{n}_{\text {zoom }}=\mathbf{2 0} \\
2 \times \text { route }_{\min } \\
\sum_{o}^{i} t_{i}=500^{\circ}\end{array}$ & $0.93 \Uparrow$ & $0.88 \Uparrow$ & 0.91 & $0.88 \Downarrow$ \\
\hline 5 & $\begin{array}{l}d_{L O C}=10 \mathrm{~m} \\
d_{L N V}=5 \mathrm{~m} \\
d_{D M}=25^{\circ} \\
d_{D A}=25^{\circ}\end{array}$ & $\begin{array}{l}n_{\text {pan }}=25 \\
n_{\text {zoom }}=10 \\
3 \times \text { route }_{\text {min }} \\
\sum_{o}^{i} \boldsymbol{t}_{\boldsymbol{i}}=\mathbf{6 0 0}^{\circ}\end{array}$ & $0.89 \Downarrow$ & $0.88 \Uparrow$ & 0.91 & $0.88 \Downarrow$ \\
\hline
\end{tabular}




\section{DISCUSSION AND LIMITATIONS}

\section{Test and Task Difficulty}

Similar item difficulties and skewness of the score data $O N M R_{v}$ were found for both locations Senden and Dülmen. Slight differences in the results for the individual items can be explained by differences in the characteristics of the task locations: More path intersections and more objects on a smaller scale might increase the difficulty due to increasing options for solving a task, but might also facilitate orientation due to the existence of several landmarks.

Overall, the range of different task difficulties ensured enough easy tasks to motivate students, but still sufficiently intermediate and harder ones to distinguish performances among stronger navigational map readers.

\section{Item-Total Correlation and Cronbach's Alpha}

Several tasks of OriGami NMR Test show a satisfactory item-total correlation. However, a few show an item-total correlation less than 0.3 - especially some Direction Tasks and some LNVtasks, predominantly for the Location Dülmen. These results are also represented in the differences of the Cronbach's Alphas: We observed acceptable Cronbach's Alphas for both locations - except from the Cronbach's Alphas for the Direction Tasks of Dülmen that are unsatisfactory low and may represent a low internal consistency (correlation) of the items.

The reason for low item-total correlations can be found in the participants' task solutions. For low values, we expect only slight or no differences in the task performances of strong and weak navigational map readers. For negative values, we expect stronger performances for weak than for strong navigational map readers.

The low item-total correlations for task LNV1 illustrates this interpretation: Some children passed the flag destination and came back to it, other children passed it and chose a wrong destination for the flag. In both groups, several children recognized their mistake afterwards and explained it to the experimenter: Since it was the initial task they were not familiar with the scale and therefore surprised that the flag was located close to the starting point. This initial-scale-problem could be observed for weak and strong navigational map readers equally which might explain the low item-total correlations.

Moreover, we saw that Direction Tasks are less helpful to distinguish between strong and weak navigational map readers than Location Tasks - especially for the location Dülmen. Two possible reasons can be figured out: (1.) The Direction Tasks were presented in a package of four tasks (one task for each cardinal direction) which made it easy to derive a task solution from the former task, and (2.) these tasks are also solvable without transforming directions in the mind (i.e. just by turning to objects the arrow on the map is pointing at).

\section{Split-Half Reliability and Validation}

We observed satisfactory split-half reliability values as well as adequate validity coefficients for both locations. Reasons for location-dependent differences were already discussed above. Strong results were observed for the Location Tasks - with no noticeable effect when including the Direction Tasks. The sensitivity analysis revealed that also changes in the thresholds defining the score categorization can go along with similar satisfactory results.

The reliability estimations were based on assumptions. Since the split-half reliability values are higher than the Cronbach's Alpha values, there is again a need for conducting a factor analysis 
with a larger data sample in order to find possible reason for this difference. Nevertheless, the presented results on the reliability revealed the potential of OriGami NMR Test to measure in a reliable and accurate way.

The satisfactory validation against the expert scoring allows us to confirm the intended test score interpretation. Therefore, our interpretation of $O N M R_{v}$ can be formulated as follows: $O N M R_{v}$ captures a child's spatial NMR competencies from a teacher's perspective and allows us to compare the competencies of children or to evaluate the learning progress of an individual child at a specific location.

\section{Process Data}

Including the process data for determining the $O N M R_{v}$ score led to slight changes in the score itself, the task difficulties, the item-total correlations, Cronbach's Alpha, split-half reliability, as well as the validation against the expert judgements. What we could not find is a clear improvement or deterioration of OriGami NMR Test when including process data. We assume that we need to examine for each task individually whether the inclusion improves the test or not. Two examples illustrate this assumption:

(1.) LNV4 senden was solved correctly by nearly half of the participants. During the experiment we witnessed that a lot of children solving this task correctly by trial-and-error. Since the destination of the flag was unique regarding the shape of a nearby object, the children recognized the destination when seeing it in their environment regardless of whether they expected the destination at that location or not - in other words: It is possible that weak navigational map reader with a trial-and-error solution solved this task correctly. This would explain the low item-total correlation for this specific task. The only difference between strong and weak navigational map readers then would be the length of the route walked (process data): Some children took longer routes than others. The longer route possibly goes along with a trial-and-error solution which would explain the increase in the task difficulty and item-total correlation when including process data into the scoring process.

(2.) For LOC4senden the opposite is the case: Including process data for this specific task leads to a decrease in the task difficulty and in the item-total correlation. Possibly, some strong navigational map readers panned and zoomed the map more often to be sure to choose the correct answer or some weak map readers chose the solution by recognizing the unique spot on the map without checking their solution for a second time via panning and zooming. 


\section{CONCLUSION}

We developed OriGami NMR Test - a digitally-supported real-world test for measuring NMR competencies in primary school children at the age from 6 to 10. The Location and Direction Tasks of the test focus on configurational and directional information and therefore on the spatial aspects of NMR. With the $O N M R_{v}$ scoring of the test, we can distinguish between strong and weak navigational map readers for a certain location or measure the learning progress of an individual map reader. Since the test has no norm-reference, it is not intended to be used for evaluating the performance of an individual against a norm group.

We evaluated OriGami NMR Test for two exemplary locations by analyzing the test items, estimating the reliability of the test, and validating the total test score against an expert judgement on the students' NMR competencies. For these two locations and the intended test score interpretation, OriGami NMR Test is shown to be reliable and valid. However, these results are based on different assumptions which need to be factor analytically proven with a larger sample size. A factor analysis would also help to decide on the dropping of individual tasks and on the method to estimate the reliability.

All suggested tasks (Location and Direction Tasks) as well as all kind of data (accuracy and process data) are - taken together - helpful to learn more on the NMR competencies of the participants. However, the results show that it is sufficient to focus on process data only when it improves the item-total correlation of the individual task. Moreover, since we aim to reduce our test to the most expressive tasks (to prevent long test durations) and since the Direction Tasks were not as successful as expected, it is sufficient and useful to reduce the test to the Location Tasks.

We offer a test design that is applicable at different locations: OriGami NMR Test is implemented in the location-based GeoGame OriGami which allows the reader to create and conduct the test for any location. We offer an R-Script that automatically measures and scores the participants' performances and evaluates the test and its items for a new location. Moreover, the R-Script helps the reader to determine two comparable test-halves which can be used as pre- and posttests for analyzing the learning progress of NMR trainings.

Future work on NMR tests should also consider virtual reality (VR) solutions to avoid locationdependent test scores and the need for re-evaluating the test for new locations. Moreover, VR would facilitate the creation of ideal worlds that do not include unique locations and therefore prevent trial-and-error solutions. 


\section{LITERATURE}

Aretz, A. J., \& Wickens, C. D. (1992). The Mental Rotation of Map Displays. Human Performance, 5(4), 303-328.

Baker, T. R., Battersby, S., Bednarz, S. W., Bodzin, A. M., Kolvoord, B., Moore, S., Sinton, D. \& Uttal, D. (2015). A Research Agenda for Geospatial Technologies and Learning. Journal of Geography, 114(3), 118-130.

Bartoschek, T., Schwering, A., Li, R., Münzer, S., \& Carlos, V. (2018). OriGami - A Mobile Geogame for Spatial Literacy. In Geogames and Geoplay, 37-62. Springer, Cham.

Bühner, M. (2011). Einführung in die Test- und Fragebogenkonstruktion [Introduction to the design of tests and questionnaires]. Pearson, Hallbergsmoos.

CDC-HKEAA - Curriculum Development Council \& The Honk Kong Examinations and Assessment Authority (2017). Geography. Curriculum and Assessment Guide (Secondary 4 -6). https://www.edb.gov.hk/attachment/en/curriculum-development/kla/pshe/ Geog_C\&A_Guide_e-Nov_2017_clean_ok.pdf

Dabbs Jr, J. M., Chang, E. L., Strong, R. A., \& Milun, R. (1998). Spatial Ability, Navigation Strategy, and Geographic Knowledge Among Men and Women. Evolution and Human Behavior, 19(2), 89-98.

Field, K., O'Brien, J., \& Beale, L. (2011). Paper Maps or GPS? Exploring Differences in Wayfinding Behaviour and Spatial Knowledge Acquisition. In Proceedings of the 25th International Cartographic Conference; Paris.

Fu, Z., Tian, Z., Xu, Y., \& Qiao, C. (2016). A Two-Step Clustering Approach to Extract Locations from Individual GPS Trajectory Data. ISPRS International Journal of Geo-Information, 5(10), 166.

DGfG - Deutsche Gesellschaft für Geographie (2012). Educational Standards in Geography for the Intermediate School Certificate. https://vgdh.geographie.de/wp-content/docs/2014/ 10/geography_education.pdf

Hemmer, I., Hemmer, M., Obermaier, G., \& Uphues, R. (2008). Räumliche Orientierung - Eine empirische Untersuchung zur Relevanz des Kompetenzbereichs aus der Perspektive von Gesellschaft und Experten [Spatial orientation - An Empirical Study of the Relevance of the Competence Area from the Perspective of Society and Experts]. Geographie und ihre Didaktik, 36, 17-32.

Hemmer, I., Hemmer, M., Kruschel, K., Neidhardt, E., Obermaier, G., \& Uphues, R. (2013). Which Children Can Find a Way Through a Strange Town Using a Streetmap? - Results of an Empirical Study on Children's Orientation Competence. International Research in Geographical and Environmental Education, 22(1), 23-40.

Hergan, I., \& Umek, M. (2017). Comparison of Children's Wayfinding, Using Paper Map and Mobile Navigation. International Research in Geographical and Environmental Education, 26(2), 91-106.

Hoffmann, T. (1999). The Meanings of Competency. Journal of European Industrial Training, 23(6), 275-285.

Hurst, P., \& Clough, P. (2013). Will We Be Lost Without Paper Maps in the Digital Age? Journal of Information Science, 39(1), 48-60.

Ishikawa, T., \& Kastens, K. A. (2005). Why Some Students Have Trouble with Maps and Other Spatial Representations. Journal of Geoscience Education, 53(2), 184-197.

Ishikawa, T. (2019). Satellite Navigation and Geospatial Awareness: Long-Term Effects of Using Navigation Tools on Wayfinding and Spatial Orientation. The Professional Geographer, 71(2), 197-209. 
Kozhevnikov, M., \& Hegarty, M. (2001). A Dissociation Between Object Manipulation Spatial Ability and Spatial Orientation Ability. Memory \& Cognition, 29(5), 745-756.

Liben, L. S., \& Myers, L. J. (2007). Developmental Changes in Children's Understanding of Maps: What, When, and How? In J.M. Plumert \& J.P. Spencer (Eds.), The Emerging Spatial Mind, 193-218. Hove: Psychology Press Ltd.

Lobben, A. K. (2004). Tasks, Strategies, and Cognitive Processes Associated with Navigational Map Reading: A Review Perspective. The Professional Geographer, 56(2), 270-281.

Lobben, A. K. (2007). Navigational Map Reading: Predicting Performance and Identifying Relative Influence of Map-Related Abilities. Annals of the Association of American Geographers, 97(1), 64-85.

Ministry of Education NZ (2007). The New Zealand Curriculum for English-medium teaching and learning in years 1 - 13. https://nzcurriculum.tki.org.nz/content/download/1108/11989/ file/NZ\%20Curriculum\%20Web.pdf

Moosbrugger, H., \& Kelava, A. (2020). Testtheorie und Fragebogenkonstruktion [Test Theory and Design of Questionnaires]. Springer, Berlin.

Münzer, S., Zimmer, H. D., Schwalm, M., Baus, J., \& Aslan, I. (2006). Computer-Assisted Navigation and the Acquisition of Route and Survey Knowledge. Journal of Environmental Psychology, 26(4), 300-308.

NRC - National Research Council (2006). Learning to Think Spatially. Washington, DC: The National Academies Press.

Presson, C. (1982): The Development of Map-reading Skills. Wiley on behalf of the Society for Research in Child Development, 53(1), 196-199.

Speake, J., \& Axon, S. (2012). "I Never Use 'Maps' Anymore”: Engaging with Sat Nav Technologies and the Implications for Cartographic Literacy and Spatial Awareness. The Cartographic Journal, 49(4), 326-336. 